\title{
THE IMPACT OF TRADITIONAL LAND USE MANAGEMENT ON SOIL QUALITY IN NORTHEASTERN HIMALAYAS (INDIA)
}

\author{
${ }^{1}$ Rain Forest Research Institute \\ Indian Council of Forestry Research and Education \\ 785001 Jorhat, Assam: India \\ e-mail: gaurav.mishra215@gmail.com \\ ${ }^{2}$ Department of Physical Geography \\ University of Trier \\ 54296 Trier: Germany \\ ${ }^{3}$ Soil Erosion and Degradation Research Group \\ Department of Geography, University of Valencia \\ Blasco Ibàñez, 28, 46010 Valencia: Spain \\ e-mail: jesus.rodrigo@uv.es (corresponding author)
}

Gaurav Mishra' ${ }^{1}$ - Jesús Rodrigo-Comino²,3

\begin{abstract}
In the Northeast Himalayas (NEH) region, four major conventional land-use types are forest, Jhum lands, fallow Jhum lands and plantations, but little is known about their sustainability and responses to changes. We collected soil samples at two uniform depths $(0-15$ and $15-30 \mathrm{~cm})$ from the Zunheboto district of Nagaland (India). The dataset was statistically analyzed by conducting an ANOVA-one way, principal component analysis (PCA) and calculating an additive soil quality index (SQla). Our results confirmed that sand content, bulk density (BD), porosity, soil organic carbon (SOC), cation exchange capacity (CEC), exchangeable calcium and potassium showed significant statistical differences among soil depths depending on the land use management. PCA results showed that soil texture, BD, porosity, SOC and exchangeable cations could be considered the major indicators to define soil quality. After estimating the SQla, Jhum soils showed the highest values at the surface, while at $15-30 \mathrm{~cm}$ soil depth, fallow Jhum soils phase showed the highest ones. The conversion from natural forest to plantation does not hamper the SQ, but their conversion into Jhum may even increase it, for a shorter duration. However, after 1-2 year of cultivation and conversion from Jhum into fallow Jhum land, soil quality could be reduced.
\end{abstract}

\section{Key words}

land use management - Forests - cropland $\bullet$ soil quality - Northeast Himalayas 


\section{Introduction}

The topic of human impact on soil quality in cultivated mountainous areas in non-developing regions using traditional methods since decades to subsist by changing the land use types is poorly studied. An example of this type of areas is the North-Eastern Hilly region of India (NEH). The NEH is one of the twelve biodiversity hot spots in the world due to its geographical position, favourable climate and biodiversity richness (Choudhury et al., 2016). It is covered by forests (65\%), agricultural land (16\%) and fallow lands (Saha et al., 2012). Shifting cultivation (locally called as Jhum) is one of the major sources of livelihood for most of the tribal farmers in NEH (Yadav, 2013). Approximately, $86 \%$ of the total cultivated area of $\mathrm{NEH}$ is under the practice of Jhum cultivation, with a maximum extension in the state of Nagaland (Patel et al., 2013). In the last few decades, Jhum cultivation led to a rapid change in land uses in Nagaland (Chase \& Singh, 2014; Sulieman et al., 2018), as the fallow period is decreasing, due to increase in population and food demand.

In other regions cultivated by this traditional farming, it was noted that intensive crop cultivation with no external input for 2-4 years after slash and burn of native forest are causing a significant decline in soil quality. For example, Handayani (2004) reported that cultivation after the clearance of forest in Sumatra resulted in the loss of organic matter (OM) content and reduction in labile C pools, declining subsequently biological activity. Continuous cultivation in deforested areas reduces soil cation exchange capacity (CEC) (Mulugeta et al., 2005; Nega \& Heluf, 2009). Intensive farming and site-specific changes in land-use patterns of the deforested areas may lead to various deleterious effects such as soil erosion, biodiversity loss, acidification, soil compaction, desertification and climate change (Salehi et al., 2008; Vorlaufer et al., 2017; Norman, 2020). Recently, studies on the effects of forest conversion to temporary agricultural lands on the dynamics of organic $C$ stock and soil health are currently receiving the attention of policymakers and forest managers (IPCC, 2007). Some reports suggests that shifting fallows after 2-3 year intensive cropping acts as a sink of soil organic $C$ and soil organic $C$ content increases with increasing the age of fallows (Ramakrishnan \& Toky, 1981; Arunachalam, 2002; Grogan et al., 2012). However, leaving the fields abandoned for $\sim 15$ years with grasses (Imperata cylindrica) as secondary successive vegetation and resuming crop cultivation for 3-5 years did not improve soil quality (Handayani, 2004). Thus, inconsistent conclusions had been drawn from different studies, defaulting our understanding of the effects of land-use changes on soil quality and their indicators in both short and long-term periods.

Soil productivity in Jhum lands was maintained due to partially combusted biomass, associated nutrients, mainly stored in the organic form, and nutrients held in the mineral form on the exchange sites of SOC (Palm et al., 1996; Bahr et al., 2014). In the absence of vegetation, generated $\mathrm{SOC}$ remains prone to the accelerated erosion and lost during the first and second year of cropping phase. Bahr et al. (2014) reported that almost 14-20\% of SOC in topsoil layers had been lost during the cropping phase.

Moreover, studies evaluating the influence of different land-use types on soil quality under tropical and sub-humid forest conditions are also limited (Lohbeck et al., 2015; Poorter et al., 2015). Hence, it is important to evaluate the influence of land-use changes on soil quality indicators as it was reported by other studies in arid and semi-arid areas (RodrigoComino et al., 2018; Keshavarzi et al., 2020). To achieve this kind of evaluations, different multivariate statistical methods have been already developed such as principal component analysis (PCA), factor analysis (FA) or correlation analysis (CA), which are mainly used to segregate soil parameters and environmental plot characteristics contributing more towards observed soil quality (Ozan et al., 2008; Beniston et al., 2015).

Therefore, according to the mentioned lack of studies related to soil quality indicators 
after different land uses changes in conventional agricultural fields in the Himalayas, the main aims of this research were to determine: (1) if there are differences among soil properties at different depths and land use management; (2) which soil quality indicators play a key role in explaining soil quality; and, (3) to evaluate the soil quality using minimum data set (MDS) by additive index method.

\section{Materials and methods}

\section{Study area}

This study was carried out in the Zunheboto district situated in the region of Nagaland, Northeastern Himalaya (India). The district, located at $25^{\circ} 97^{\prime} \mathrm{N}, 94^{\circ} 52^{\prime} \mathrm{E}$, occupies an area of about $1255 \mathrm{~km}^{2}$ with an average elevation of about $1800 \mathrm{~m}$ asl. (Fig. 1). The average sum of annual precipitation is close to $2000 \mathrm{~mm}$, where $90 \%$ of it uses to occur from May to October. Temperature varies from a minimum of $10^{\circ} \mathrm{C}$ to maximum values of $22^{\circ} \mathrm{C}$ during the year. The majority of the forest area in the Zunheboto district is classified as montane wet temperate forest (FSI, 2009). The major tree species of this area are typically evergreen with Alnus nepalensis, Quercus sps., Magnolia champaca, Schima walichi, and Betula alniodes. Plantations were raised by the local communities as per their need or based on timber values. They mainly consist of Pine (Pinus kesiya), Hollock (Terminaliya myrocarpa), Alder (Alnus nepalensis), Gamari (Gmelina arborea) among others. In croplands, mainly upland rice (Oryza sati$v a)$ is cultivated. Maize (Zea mays), cowpea (Vigna unguiculata), Colocasia (Colocasia esculenta), chilli (Capsicum annuum), pumpkin (Cucurbita peto), and brinjal (Solanum melongena) are also usually grown. Fallow lands mainly consist of trees along with common weeds (Eluesine indica; Amaranthus viridis; Chromolaena odorata; Mimosa pudica;

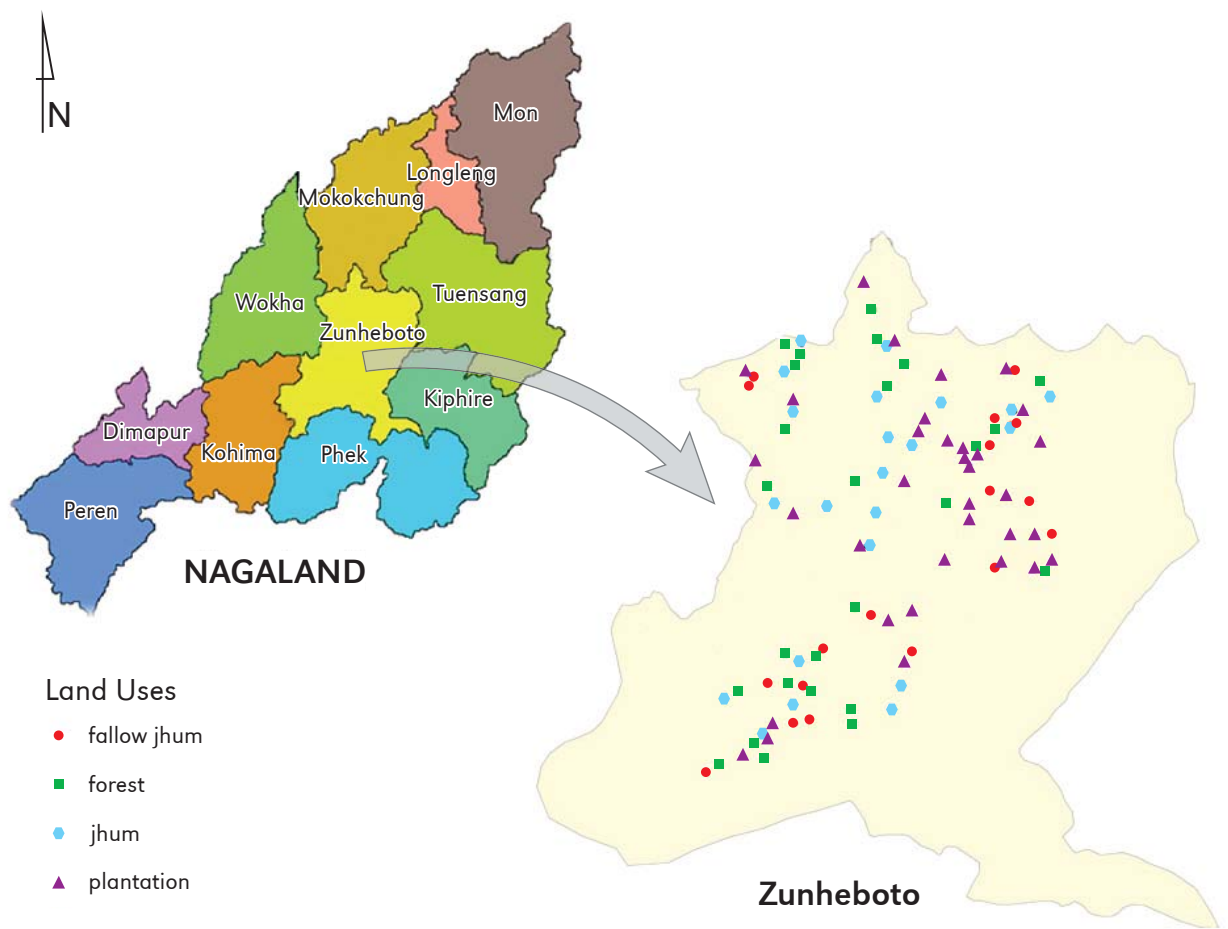

Figure 1. Study area, Zunheboto, Nagaland (India). Different colors indicate each land use (fallow Jhum, forest, Jhum and plantations, respectively) 
and Imperata cylindrica) of the region. The Jhum fields selected in this study were cropped one and fallow Jhum lands were those, which were left as fallow for more than five years, after cultivation.

\section{Soil sampling and analysis}

During 2016-17, 88 different land uses sites were selected, based on altitude, aspect and inclination gradient (Fig. 2). At each site, soil samples from two different depths were collected. Sampling depth was fixed at $0-15 \mathrm{~cm}$ and $15-30 \mathrm{~cm}$ depth, considering that 0-15 cm soil depth is more dynamic, during cropping phase. We hypothesize that as an indication of dynamic soil quality, soil properties at surface $(0-15 \mathrm{~cm})$ level can be considered vital in jhum lands, whereas the inherent quality of the soil at $15-30 \mathrm{~cm}$, could be more relevant in forests and plantations. The samples were air-dried and sieved through 2-mm sieve to exclude litter, roots and coarse particles. Soil samples were analyzed for soil texture, bulk density (BD), porosity, $\mathrm{pH}$ and soil organic carbon (SOC). The particle size distribution was obtained by the hydrometer method (Klute, 1986). BD was calculated by the core method with a copper cylinder of known volume $\left(134.16 \mathrm{~cm}^{3}\right)$ (Blake \& Hartge, 1986), meanwhile, total porosity was calculated from the bulk density values assuming the obtained particle density (USDA- NRCS, 2004). Soil pH value was determined in 1:2 soil water suspension using digital $\mathrm{pH}$-meter. Soil organic carbon (SOC) was analyzed following the method by Walkley and Black (1934).

Available phosphorus (P) was determined following the standard method of Bray and Kurtz (1945). Also, available potassium $(\mathrm{K})$, cation exchange capacity (CEC) and exchangeable cations ( $\mathrm{Ca}, \mathrm{Na}$, and $\mathrm{Mg}$ ) were estimated by $1 \mathrm{~N}$ ammonium acetate $(\mathrm{pH} 7.0)$ method (Schollenberger \& Simon, 1945; Sumner \& Miller, 1996). Exchangeable aluminium
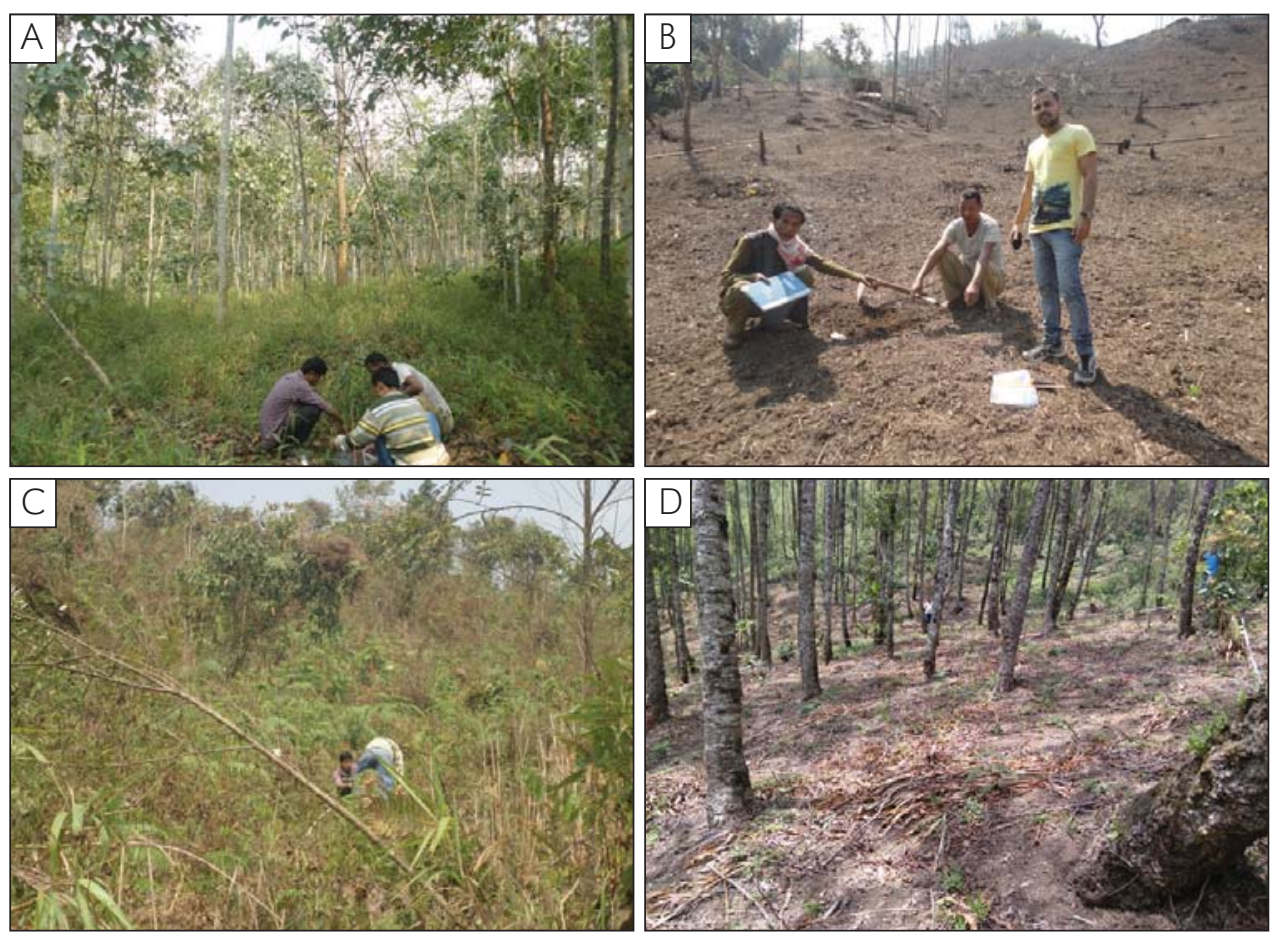

Figure 2. Soil sample collection from different land use systems (A: forest; B: Jhum; C: fallow Jhum and $\mathrm{D}$ : plantation) 
(Al) was extracted with $1 \mathrm{~N} \mathrm{KCl}$ solution and titrated with 0.1 $\mathrm{N} \mathrm{NaOH}$ (McLean, 1965).

\section{Statistical analysis}

To assess the statistical differences among soil properties under different land uses, an ANOVA-one-way test was conducted per land use and soil depth. To check the normality of the data, a Shapiro-Wilks test was also carried out. Also, the variance was checked. If they failed, a Kruskal-Wallis One Way Analysis of Variance on Ranks was performed. SigmaPlot 13.0 (Systat Inc.) was the statistical program used to analyze the data. After that, PCA was conducted using SPSS 23.0 (IBM, USA). Firstly, a Pearson correlation was conducted to assess which soil property and quality indicator could be correlated. However, as the tables are too big due to the several soil properties used, these results were not included (they can be sent after a formal request).

To identify which driving factors condition soil properties and quality indicators, PCA was carried out. With this technique, we were able to group similar variables into dimensions, without distinguishing between independent and dependent variables. The first step was to centre and normalize the variables. Then, we verified them making a Kaiser-Mayer-Olkin (KMO) test of sphericity. Moreover, an orthogonal rotation method (Varimax), and a correlation matrix, using factors with Eigenvalues $>1$ were applied. Soil properties and quality indicators used to achieve our main objective in this research should model the phenomenon pretended to be detected (relationships among them) in the closest possible way, registering specifically the most significant parameters that affect soil distribution and pedogenesis (Shukla et al., 2006). PCA was carried out for the above-mentioned different land uses plantation (P), Jhum land (crop) (J), fallow Jhum land (FJ) and forest (f) and at different depths $(0-15$ and $15-30 \mathrm{~cm})$.

\section{Soil quality index (SQI)}

The SQI was calculated using depth-wise data of all land-use sites. The SQI was calculated using an additive index approach at both the depths (Abdel Rahman et al., 2019; Mukherjee \& Lal, 2014). The transformed scores from each soil depth were added for the calculation of the additive index. The variability

Table 1. Soil properties (part 1) and comparison among different soil depths

\begin{tabular}{|l|c|c|c|c|c|c|c|c|}
\hline $\begin{array}{c}\text { Land } \\
\text { use }\end{array}$ & $\begin{array}{c}\text { Soil } \\
\text { depth }\end{array}$ & $\begin{array}{c}\text { Clay } \\
{[\%]}\end{array}$ & $\begin{array}{c}\text { Silt } \\
{[\%]}\end{array}$ & $\begin{array}{c}\text { Sand } \\
{[\%]}\end{array}$ & $\begin{array}{c}\text { BD } \\
{\left[\mathrm{g} \mathrm{cm}^{-3}\right]}\end{array}$ & $\begin{array}{c}\text { Porosity } \\
{[\%]}\end{array}$ & $\begin{array}{c}\mathrm{pH} \\
\begin{array}{c}\text { SOC } \\
{[\%]}\end{array}\end{array}$ \\
\hline P & $0-15$ & $26.6 \pm 14.4$ & $25.4 \pm 10.2$ & $48 \pm 18.5$ & $0.80 \pm 0.1$ & $69.9 \pm 4.1$ & $4.8 \pm 0.5$ & $3.2 \pm 1.1$ \\
Diff. & $15-30$ & $26.4 \pm 13.5$ & $26.5 \pm 12.0$ & $47.1 \pm 17.9$ & $0.84 \pm 0.1$ & $68.3 \pm 3.3$ & $4.6 \pm 0.4$ & $2.3 \pm 1.0$ \\
$\mathbf{p}<0.004$
\end{tabular}

* Shapiro-Wilk test failed, Kruskal-Wallis One Way Analysis of Variance on Ranks was performed; 士: Standard deviation; P: Plantation; J: Jhum; FJ: Fallow Jhum; F: Forest; BD: Bulk density; SOC: Soil organic carbon. 
of calculated SQI among land-use types was tested with one-way analysis of variance (ANOVA) followed by Duncan's test $(P<0.05)$ and the results depicted in box plots.

\section{Results}

\section{Soil properties at different depth under diverse land uses}

Soil texture was similar to all the land uses, clay content was higher than $20 \%$ and a high sand fraction (> 40\%). In Jhum lands, a significant increase was noticed in sand percentage, at $15-30 \mathrm{~cm}$ soil depth, while the fine fractions decreased. These results confirm that $\mathrm{BD}$ increases and porosity decrease at the sub-surface, making these layers heavier and defaulting water flows. A similar dynamic is also registered in fallow Jhum lands, where an increase in BD generates a decrease of porosity, showing statistical differences between soil depths $(p<0.050)$. The $\mathrm{pH}$ values do not show any differences among soil depths. On the contrary, SOC registers statistical differences among soil depths in plantation areas, fallow lands and forest. Only Jhum land shows similar values among soil depths.

Table 1 and 2 shows that plantation and forest have more homogeneous values among soil depths. In all land uses, CEC decreases from the top layer to the sub-surface one, showing a significant statistical difference in a fallow Jhum land. Also, exchangeable $\mathrm{Ca}, \mathrm{Mg}, \mathrm{Na}$ and $\mathrm{K}$ register a decrease from 0-15 cm to $15-30 \mathrm{~cm}$, showing more significant in Jhum and fallow Jhum land. The Al content increases in the plantation, Jhum and fallow Jhum land, and only decrease in forestry areas. Regarding $P$ and $K$, in cultivated areas (plantation, Jhum and fallow Jhum), a decrease from $0-15 \mathrm{~cm}$ to $15-30 \mathrm{~cm}$ can be observed. Only in the forest, soil nutrients contents ( $P$ and $\mathrm{K}$ ) increase at the sub-surface layers.

\section{Principal component analysis}

For all the land use management types, a PCA was carried out and the total variance explained after rotation sums of square loadings with the obtained components are summarized in Table 3. In general, almost all the results are

Table 2. Soil properties and comparison among different soil depths (part 2)

\begin{tabular}{|c|c|c|c|c|c|c|c|c|c|}
\hline $\begin{array}{l}\text { Land } \\
\text { use }\end{array}$ & $\begin{array}{c}\text { Soil } \\
\text { depth }\end{array}$ & $\begin{array}{c}\text { CEC } \\
{\left[\mathrm{cmol}^{-1} \mathrm{p}^{+}\right)} \\
\left.\mathrm{kg}^{-1}\right]\end{array}$ & $\begin{array}{c}\text { Ex.Ca } \\
{\left[\mathrm{cmol}^{-1} \mathrm{p}^{+}\right)} \\
\left.\mathrm{kg}^{-1}\right]\end{array}$ & $\begin{array}{c}\text { Ex. Mg } \\
{\left[\begin{array}{c}\mathrm{cmol}\left(\mathrm{p}^{+}\right) \\
\left.\mathrm{kg}^{-1}\right]\end{array}\right.}\end{array}$ & $\begin{array}{c}\text { Ex. } \mathrm{Na} \\
{\left[\mathrm{cmol}\left(\mathrm{p}^{+}\right)\right.} \\
\left.\mathrm{kg}^{-1}\right]\end{array}$ & $\begin{array}{c}\text { Ex.K } \\
{\left[\mathrm{cmol}^{-1} \mathrm{p}^{+}\right)} \\
\left.\mathrm{kg}^{-1}\right]\end{array}$ & $\begin{array}{c}\mathrm{Al} \\
{\left[\mathrm{cmol}^{-1} \mathrm{p}^{+}\right)} \\
\left.\mathrm{kg}^{-1}\right]\end{array}$ & $\begin{array}{c}\mathrm{P} \\
{[\mathrm{ppm}]}\end{array}$ & $\begin{array}{c}\mathrm{K} \\
{[\mathrm{ppm}]}\end{array}$ \\
\hline \multirow[t]{2}{*}{ P } & $0-15$ & $17.4 \pm 5.0$ & $1.9 \pm 0.4$ & $1.0 \pm 0.7$ & $3.3 \pm 3.0$ & $2.0 \pm 0.9$ & $3.9 \pm 2.7$ & $10.1 \pm 3.1$ & $50.4 \pm 15.5$ \\
\hline & $15-30$ & $13.9 \pm 4.3$ & $1.9 \pm 0.3$ & $0.8 \pm 0.6$ & $2.9 \pm 2.6$ & $1.7 \pm 0.9$ & $4.4 \pm 2.3$ & $9.4 \pm 3.9$ & $46.9 \pm 19.7$ \\
\hline Diff. & & $p<0.815^{\star}$ & $p<0.006$ & $p<0.658^{\star}$ & $p<0.338^{\star}$ & $\mathrm{p}<0.237^{\star}$ & $p<0.316^{\star}$ & $\mathrm{p}<0.129^{\star}$ & $p<0.129^{\star}$ \\
\hline \multirow[t]{2}{*}{ J } & $0-15$ & $17.5 \pm 5.5$ & $2.0 \pm 0.4$ & $0.6 \pm 0.7$ & $1.6 \pm 0.2$ & $2.0 \pm 0.8$ & $2.6 \pm 2.0$ & $11.4 \pm 4.8$ & $56.9 \pm 24.0$ \\
\hline & $15-30$ & $15.0 \pm 6.3$ & $1.7 \pm 0.5$ & $0.4 \pm 0.3$ & $1.5 \pm 0.4$ & $1.5 \pm 0.6$ & $3.9 \pm 2.1$ & $10.0 \pm 6.5$ & $50.0 \pm 32.4$ \\
\hline Diff. & & $p<0.069$ & $p<0.227$ & $p<0.275^{\star}$ & $p<0.220^{\star}$ & $\mathrm{p}<0.043$ & $p<0.071$ & $p<0.062^{\star}$ & $p<0.062^{\star}$ \\
\hline \multirow[t]{2}{*}{ FJ } & $0-15$ & $15.9 \pm 5.2$ & $2.0 \pm 0.4$ & $1.0 \pm 0.8$ & $3.5 \pm 3.0$ & $2.2 \pm 1.1$ & $3.8 \pm 2.7$ & $8.5 \pm 1.6$ & $42.5 \pm 8.2$ \\
\hline & $15-30$ & $11.6 \pm 4.0$ & $1.6 \pm 0.5$ & $0.9 \pm 0.7$ & $2.6 \pm 2.3$ & $1.5 \pm 0.6$ & $4.1 \pm 2.7$ & $8.0 \pm 1.8$ & $39.8 \pm 9.0$ \\
\hline Diff. & & $\mathrm{p}<0.010$ & $p<0.009$ & $p<0.282^{\star}$ & $p<0.824^{\star}$ & $\mathrm{p}<0.005$ & $p<0.788^{\star}$ & $p<0.352$ & $p<0.352$ \\
\hline \multirow[t]{2}{*}{$\mathrm{F}$} & $0-15$ & $12.3 \pm 3.7$ & $2.0 \pm 0.4$ & $0.8 \pm 0.4$ & $1.7 \pm 0.7$ & $1.9 \pm 0.9$ & $4.5 \pm 3.2$ & $9.1 \pm 3.3$ & $45.7 \pm 16.7$ \\
\hline & $15-30$ & $11.9 \pm 5.3$ & $1.9 \pm 0.4$ & $0.6 \pm 0.4$ & $1.7 \pm 0.8$ & $1.5 \pm 0.7$ & $4.1 \pm 2.1$ & $9.9 \pm 6.2$ & $49.4 \pm 30.9$ \\
\hline Diff. & & $p<0.203$ & $p<0.760$ & $p<0.467^{\star}$ & $p<0.083^{\star}$ & $p<0.080^{\star}$ & $\mathrm{p}<0.879^{\star}$ & $p<0.630^{\star}$ & $p<0.630$ \\
\hline
\end{tabular}

* Shapiro-Wilk test failed, Kruskal-Wallis One Way Analysis of Variance on Ranks was performed; 土: Standard deviation; P: Plantation; J: Jhum; FJ: Fallow Jhum; F: Forest; CEC: Cation-exchange capacity; Ex.Ca: exchangeable calcium; Ex. Mg: exchangeable magnesium; Ex.K: exchangeable potassium; Al: Aluminum; P: Phosphorus; K: Potassium. 
Table 3. Total variance explained after performing a rotation sum of squared loadings

\begin{tabular}{|l|c|c|c|c|c|c|c|c|}
\hline & \multicolumn{2}{|c|}{ Plantation } & \multicolumn{2}{c|}{ Jhum } & \multicolumn{2}{c|}{ Fallow Jhum } & \multicolumn{2}{c|}{ Forest } \\
\hline Component & $0-15 \mathrm{~cm}$ & $15-30 \mathrm{~cm}$ & $0-15 \mathrm{~cm}$ & $15-30 \mathrm{~cm}$ & $0-15 \mathrm{~cm}$ & $15-30 \mathrm{~cm}$ & $0-15 \mathrm{~cm}$ & $15-30 \mathrm{~cm}$ \\
\hline 1 & 18.5 & 24.6 & 27.6 & 40.1 & 21.8 & 26.7 & 16.4 & 24.1 \\
2 & 34.8 & 44.7 & 43.9 & 67.2 & 38.0 & 48.5 & 31.6 & 47.8 \\
3 & 50.8 & 63.1 & 58.9 & 86.7 & 54.1 & 66.4 & 46.7 & 69.5 \\
4 & 65.2 & 79.9 & 69.9 & 98.0 & 68.1 & 80.1 & 61.6 & 88.3 \\
5 & 75.7 & - & 80.4 & - & 80.4 & 89.1 & 72.6 & - \\
6 & 84.0 & - & - & - & - & - & 82.8 & - \\
\hline
\end{tabular}

${ }^{\star}$ Results are presented in cumulative $\%$.

Table 4. PCA (Principal component analysis) results for plantation land use

\begin{tabular}{|c|c|c|c|c|c|c|c|c|c|c|}
\hline & \multicolumn{6}{|c|}{$0-15 \mathrm{~cm}$} & \multicolumn{4}{|c|}{$15-30 \mathrm{~cm}$} \\
\hline Variables & 1 & 2 & 3 & 4 & 5 & 6 & 1 & 2 & 3 & 4 \\
\hline Clay & -0.86 & -0.26 & -0.03 & 0.01 & -0.01 & -0.06 & -0.45 & 0.69 & -0.23 & -0.12 \\
\hline Sand & 0.73 & 0.39 & -0.01 & 0.08 & -0.45 & -0.06 & 0.97 & -0.19 & 0.06 & 0.08 \\
\hline Silt & -0.11 & -0.34 & 0.07 & -0.15 & 0.83 & 0.19 & -0.85 & -0.30 & 0.10 & 0.00 \\
\hline $\mathrm{BD}$ & 0.18 & 0.95 & 0.07 & 0.10 & -0.14 & 0.02 & -0.10 & -0.94 & -0.03 & -0.11 \\
\hline Porosity & -0.19 & -0.95 & -0.07 & -0.10 & 0.14 & -0.02 & 0.09 & 0.94 & 0.02 & 0.12 \\
\hline $\mathrm{pH}$ & 0.78 & 0.34 & -0.29 & 0.03 & 0.11 & 0.08 & 0.32 & -0.36 & -0.10 & 0.82 \\
\hline SOC & 0.19 & -0.28 & 0.38 & 0.51 & 0.10 & 0.38 & 0.15 & -0.13 & 0.88 & 0.38 \\
\hline CEC & -0.13 & 0.00 & 0.34 & 0.32 & 0.70 & -0.25 & 0.15 & -0.13 & 0.88 & 0.38 \\
\hline ex.Ca & -0.02 & 0.06 & -0.16 & 0.14 & 0.02 & 0.92 & -0.20 & 0.38 & 0.43 & 0.77 \\
\hline ex.Mg & -0.01 & 0.11 & -0.17 & 0.86 & 0.01 & -0.13 & 0.84 & 0.02 & 0.35 & 0.10 \\
\hline ex. $\mathrm{Na}$ & 0.02 & 0.09 & 0.09 & 0.85 & -0.07 & 0.32 & -0.05 & -0.01 & 0.28 & 0.93 \\
\hline ex.K & 0.36 & 0.23 & -0.36 & 0.51 & 0.28 & 0.09 & -0.72 & -0.03 & 0.63 & 0.14 \\
\hline $\mathrm{Al}$ & -0.78 & 0.19 & -0.02 & -0.08 & 0.18 & 0.07 & 0.54 & -0.34 & 0.30 & -0.43 \\
\hline$P$ & -0.06 & 0.07 & 0.96 & -0.04 & 0.11 & -0.08 & 0.01 & 0.87 & -0.26 & -0.37 \\
\hline K & -0.06 & 0.07 & 0.96 & -0.04 & 0.11 & -0.08 & 0.02 & -0.04 & 0.83 & -0.15 \\
\hline \multicolumn{7}{|c|}{ Component 1: Clay, sand, $\mathrm{pH}, \mathrm{Al}$} & \multicolumn{4}{|c|}{ Component 1: Sand, silt, ex. Mg, ex. K, A } \\
\hline \multicolumn{7}{|c|}{ Component 2: BD, Porosity } & \multicolumn{4}{|c|}{ Component 2: Clay, BD, porosity, $\mathrm{P}$} \\
\hline \multicolumn{7}{|c|}{ Component 3: P, K. } & \multicolumn{4}{|c|}{ Component 3: SOC, CEC, ex. K, K } \\
\hline \multicolumn{7}{|c|}{ Component 4: SOC, ex. Mg, ex. $\mathrm{Na}$, ex, K } & \multirow{3}{*}{\multicolumn{4}{|c|}{ Component 4: pH, ex. Ca, ex. $\mathrm{Na}, \mathrm{Al}$}} \\
\hline \multicolumn{7}{|c|}{ Component 5: Silt, CEC } & & & & \\
\hline \multicolumn{7}{|c|}{ Component 6: SOC, ex. Ca } & & & & \\
\hline
\end{tabular}

*Selected values correspond to the values $>0.5$ or $>-0.5$. BD: Bulk density; SOC: Soil organic carbon; CEC: Cation-exchange capacity; Ex.Ca: exchangeable calcium; Ex. Mg: exchangeable magnesium; Ex.K: exchangeable potassium; Al: Aluminum; P: Phosphorus; K: Potassium.

*Bold and underlined factors identified the indicators retained in the MDS and used to calculate SQI. 
close to $80 \%$, showing high confidence in the obtained results. In plantation land use, total variance in surface (84.0\%) and sub-surface $(79.9 \%)$ soils is explained by six and four components, respectively. At $0-15 \mathrm{~cm}$ depth, clay $(-0.86)$ was highly weighed property in Principal component (PC) 1, so it was selected as an SQ indicator, due to its high weight in PCA analysis (Tab. 4). Similarly, BD, P, ex. Mg, silt and ex. Ca were selected as SQ indicator from different PCs at 0-15 cm soil depth. At 15-30 cm, ex. Mg, porosity, SOC and ex. $\mathrm{Na}$ was the selected soil SQ indicator in plantation soil, after PCA. In Jhum lands, sand, SOC, porosity, ex. $\mathrm{Mg}$ and ex Ca were the selected and $\mathrm{K}$, porosity, ex $\mathrm{Mg}$ and ex. K was chosen as SQ indicator for 0-15 and $15-30 \mathrm{~cm}$ depth, respectively (Tab. 5). However, in fallow Jhum lands, the selected parameters were, CEC, silt, clay, ex. $\mathrm{Na}$ and ex $\mathrm{Na}$ at $0-15 \mathrm{~cm}$ and for $15-30 \mathrm{~cm}$ ex. Mg, porosity, SOC, $\mathrm{pH}$ and Al were considered as SQ indicator (Tab. 6). Finally in forestry areas, for $0-15 \mathrm{~cm}$, SOC, clay, BD, ex. K, silt and ex. Na was selected as SQ indicator (Tab. 7). Further at $15-30 \mathrm{~cm}$, ex. K, porosity, SOC and ex. Na were highly weighted soil properties after PCA, so they are also selected.

Table 5. PCA (Principal component analysis) results for Jhum land use

\begin{tabular}{|c|c|c|c|c|c|c|c|c|c|}
\hline & \multicolumn{5}{|c|}{$0-15 \mathrm{~cm}$} & \multicolumn{4}{|c|}{$15-30 \mathrm{~cm}$} \\
\hline Variables & 1 & 2 & 3 & 4 & 5 & 1 & 2 & 3 & 4 \\
\hline Clay & -0.75 & -0.10 & 0.16 & 0.03 & -0.31 & 0.97 & -0.03 & -0.13 & 0.20 \\
\hline Sand & 0.95 & 0.07 & -0.04 & 0.02 & 0.14 & -0.99 & -0.02 & 0.10 & -0.10 \\
\hline Silt & -0.81 & -0.02 & -0.12 & -0.08 & 0.10 & 0.99 & 0.08 & -0.05 & -0.03 \\
\hline $\mathrm{BD}$ & 0.09 & -0.04 & -0.97 & -0.08 & -0.12 & 0.08 & -0.95 & 0.30 & -0.05 \\
\hline Porosity & -0.09 & 0.04 & 0.97 & 0.08 & 0.12 & -0.08 & 0.95 & -0.30 & 0.05 \\
\hline $\mathrm{pH}$ & 0.81 & 0.30 & -0.11 & 0.13 & -0.04 & -0.66 & -0.37 & 0.61 & 0.23 \\
\hline SOC & 0.22 & 0.90 & 0.06 & 0.19 & 0.23 & 0.38 & 0.88 & 0.15 & -0.23 \\
\hline CEC & 0.22 & 0.90 & 0.06 & 0.19 & 0.23 & 0.38 & 0.88 & 0.15 & -0.23 \\
\hline ex.Ca & -0.35 & 0.38 & 0.26 & -0.07 & 0.74 & 0.91 & 0.37 & 0.12 & 0.16 \\
\hline ex.Mg & 0.22 & 0.06 & -0.06 & 0.85 & 0.01 & 0.02 & -0.21 & 0.97 & 0.11 \\
\hline ex. $\mathrm{Na}$ & -0.49 & -0.31 & 0.05 & 0.53 & 0.11 & 0.03 & -0.03 & 0.96 & -0.11 \\
\hline ex.K & 0.03 & 0.24 & 0.20 & 0.67 & 0.00 & 0.20 & -0.12 & -0.06 & 0.96 \\
\hline $\mathrm{Al}$ & 0.73 & -0.20 & -0.40 & 0.15 & 0.08 & -0.71 & 0.14 & -0.10 & -0.60 \\
\hline P & -0.29 & 0.60 & 0.00 & -0.26 & -0.46 & 0.16 & -0.58 & -0.64 & 0.37 \\
\hline K & 0.44 & 0.13 & 0.14 & 0.06 & 0.74 & 0.99 & 0.09 & 0.09 & 0.10 \\
\hline \multicolumn{6}{|c|}{ Component 1: Clay, sand, silt, $\mathrm{pH}, \mathrm{Al}, \mathrm{K}$} & \multicolumn{4}{|c|}{$\begin{array}{l}\text { Component 1: Clay, sand, silt, pH, ex. Ca, } \\
\text { Al, K }\end{array}$} \\
\hline \multicolumn{6}{|c|}{ Component 2: SOC, CEC, P } & \multicolumn{4}{|c|}{ Component 2: BD, porosity, SOC, CEC } \\
\hline \multicolumn{6}{|c|}{ Component 3: BD, porosity } & \multicolumn{4}{|c|}{ Component 3: pH, ex. Mg, ex. $\mathrm{Na}, \mathrm{P}$} \\
\hline \multicolumn{6}{|c|}{ Component 4: ex. Mg, ex. $\mathrm{Na}$, ex, $\mathrm{K}$} & \multirow{2}{*}{\multicolumn{4}{|c|}{ Component 4: pH, ex. K, Al }} \\
\hline \multicolumn{6}{|c|}{ Component 5: ex. Ca, K } & & & & \\
\hline
\end{tabular}

*Selected values correspond to the values $>0.5$ or $>0.5$. BD: Bulk density; SOC: Soil organic carbon; CEC: Cation-exchange capacity; Ex.Ca: exchangeable calcium; Ex. Mg: exchangeable magnesium; Ex.K: exchangeable potassium; Al: Aluminum; P: Phosphorus; K: Potassium.

*Bold and underlined factors identified the indicators retained in the MDS and used to calculate SQI. 
Table 6. PCA (Principal component analysis) results for fallow Jhum land use

\begin{tabular}{|c|c|c|c|c|c|c|c|c|c|c|}
\hline & \multicolumn{5}{|c|}{$0-15 \mathrm{~cm}$} & \multicolumn{5}{|c|}{$15-30 \mathrm{~cm}$} \\
\hline Variables & 1 & 2 & 3 & 4 & 5 & 1 & 2 & 3 & 4 & 5 \\
\hline Clay & 0.07 & 0.15 & 0.89 & 0.15 & 0.14 & 0.90 & -0.04 & 0.20 & -0.17 & -0.11 \\
\hline Sand & -0.03 & 0.63 & -0.65 & -0.01 & -0.17 & -0.37 & 0.77 & 0.00 & 0.38 & -0.21 \\
\hline Silt & -0.03 & -0.87 & 0.03 & -0.11 & 0.09 & -0.17 & -0.84 & -0.12 & -0.32 & 0.31 \\
\hline $\mathrm{BD}$ & -0.54 & -0.65 & -0.09 & 0.30 & 0.10 & 0.09 & -0.91 & -0.08 & 0.19 & -0.19 \\
\hline Porosity & 0.54 & 0.65 & 0.09 & -0.30 & -0.10 & -0.09 & 0.92 & 0.07 & -0.20 & 0.17 \\
\hline $\mathrm{pH}$ & -0.44 & 0.00 & -0.66 & 0.31 & 0.21 & 0.19 & -0.01 & -0.14 & 0.86 & 0.12 \\
\hline SOC & 0.94 & 0.13 & 0.15 & -0.01 & 0.00 & 0.02 & 0.05 & 0.97 & -0.17 & 0.02 \\
\hline CEC & 0.94 & 0.13 & 0.15 & -0.01 & 0.00 & 0.02 & 0.05 & 0.97 & -0.17 & 0.02 \\
\hline ex.Ca & 0.46 & 0.51 & 0.30 & 0.14 & -0.39 & 0.47 & 0.45 & 0.62 & 0.08 & -0.14 \\
\hline ex.Mg & -0.13 & -0.09 & 0.05 & -0.05 & 0.81 & $\underline{0.83}$ & -0.19 & -0.12 & 0.26 & 0.10 \\
\hline ex. $\mathrm{Na}$ & -0.13 & -0.05 & 0.12 & 0.92 & -0.16 & 0.80 & 0.12 & 0.03 & -0.13 & 0.40 \\
\hline ex.K & -0.43 & -0.10 & 0.12 & 0.62 & 0.47 & 0.82 & -0.23 & -0.07 & 0.18 & -0.02 \\
\hline $\mathrm{Al}$ & 0.31 & -0.27 & -0.19 & -0.21 & 0.62 & 0.12 & 0.02 & 0.02 & 0.35 & 0.90 \\
\hline P & 0.12 & -0.03 & 0.66 & -0.02 & -0.46 & 0.32 & -0.02 & 0.32 & -0.79 & -0.32 \\
\hline K & 0.41 & 0.11 & -0.32 & 0.71 & -0.26 & 0.80 & 0.10 & 0.45 & -0.17 & -0.04 \\
\hline \multicolumn{6}{|c|}{ Component 1: BD, porosity, pH, SOC, CEC } & \multicolumn{5}{|c|}{ Component 1: Clay, ex. Ca, ex. Mg, ex. K, K } \\
\hline \multicolumn{6}{|c|}{ Component 2: Sand, silt, BD, porosity, ex. Ca } & \multicolumn{5}{|c|}{ Component 2: Sand, silt, BD, porosity, ex. Ca } \\
\hline \multicolumn{6}{|c|}{ Component 3: Clay, sand, pH, P } & \multicolumn{5}{|c|}{ Component 3: SOC, CEC, ex. Ca } \\
\hline \multicolumn{6}{|c|}{ Component 4: ex. $\mathrm{Na}, \mathrm{ex}, \mathrm{K}, \mathrm{K}$} & \multicolumn{5}{|c|}{ Component 4: $\mathrm{pH}, \mathrm{P}$} \\
\hline \multicolumn{6}{|c|}{ Component 5: ex. Mg, Al, K } & \multicolumn{5}{|c|}{ Component 5: ex. $\mathrm{Na}$, ex. $\mathrm{Al}$} \\
\hline
\end{tabular}

${ }^{\star}$ Selected values correspond to the values $>0.5$ or $>-0.5$. BD: Bulk density; SOC: Soil organic carbon; CEC: Cation-exchange capacity; Ex.Ca: exchangeable calcium; Ex. Mg: exchangeable magnesium; Ex.K: exchangeable potassium; Al: Aluminum; P: Phosphorus; K: Potassium.

${ }^{*}$ Bold and underlined factors identified the indicators retained in the MDS and used to calculate SQI.

\section{Soil quality under different land uses}

In Plantation, for 0-15 cm soil depth clay, BD, P, ex. Mg, silt and ex. Ca from PC 1, PC2, PC3, PC4, PC5 and PC6, while for $15-30 \mathrm{~cm}$, ex. $\mathrm{Mg}$, porosity, SOC and ex. Na are selected as indicators for MDS. In Jhum lands, sand, SOC, porosity, ex. Mg, ex. Ca in 0-15 cm and K, porosity, ex. Mg, ex. Kat $15-30 \mathrm{~cm}$ is considered as indicators. For fallow Jhum land and forest soil, selected indicators are mentioned in their respective Tables 6 and 7. Using these MDS, additive SQIs for the soils under four land-use types at two different depths (0-15 and $15-30 \mathrm{~cm}$ ), were calculated. The detailed information of the intra-variations of each land related to $\mathrm{SQI}$ is provided in Figure 3 . In Table 8, the results of additive SQI are summarized and it is found that land uses have a significant effect on soil quality at both depths. For the surface soil $(0-15 \mathrm{~cm})$ layer, additive SQI is in the order of Jhum (3.51) > plantation (3.29) > forest (3.27) > fallow Jhum land (2.13). Moreover, fallow Jhum lands have significantly lower $(F=24.445, p=0.00)$ value of SQI than other land uses. However, at $15-30 \mathrm{~cm}$ layer, the trend is different. Fallow Jhum lands have significantly the highest $(F=15.585, p=0.00)$ value of additive SQI (3.01), followed by Jhum (2.52). Although, forest (2.18) and plantations (2.15) soils have a more or less similar value of SQI. 
Table 7. PCA (Principal component analysis) results for forest land use

\begin{tabular}{|c|c|c|c|c|c|c|c|c|c|c|}
\hline & \multicolumn{6}{|c|}{$0-15 \mathrm{~cm}$} & \multicolumn{4}{|c|}{$15-30 \mathrm{~cm}$} \\
\hline Variables & 1 & 2 & 3 & 4 & 5 & 6 & 1 & 2 & 3 & 4 \\
\hline Clay & -0.01 & -0.92 & 0.13 & -0.16 & 0.16 & -0.06 & -0.45 & 0.69 & -0.23 & -0.12 \\
\hline Sand & -0.08 & 0.77 & -0.02 & 0.10 & 0.54 & 0.00 & 0.97 & -0.19 & 0.06 & 0.08 \\
\hline Silt & 0.14 & 0.00 & -0.13 & 0.03 & -0.88 & 0.05 & -0.85 & -0.30 & 0.10 & 0.00 \\
\hline$B D$ & 0.05 & -0.05 & 0.99 & 0.01 & 0.05 & 0.00 & -0.10 & -0.94 & -0.03 & -0.11 \\
\hline Porosity & -0.05 & 0.05 & -0.99 & -0.01 & -0.05 & 0.00 & 0.09 & 0.94 & 0.02 & 0.12 \\
\hline $\mathrm{pH}$ & -0.06 & 0.60 & 0.25 & 0.40 & 0.06 & 0.49 & 0.32 & -0.36 & -0.10 & 0.82 \\
\hline SOC & 0.95 & -0.04 & 0.10 & -0.03 & -0.13 & -0.14 & 0.15 & -0.13 & 0.88 & 0.38 \\
\hline CEC & 0.95 & -0.04 & 0.10 & -0.03 & -0.13 & -0.14 & 0.15 & -0.13 & 0.88 & 0.38 \\
\hline ex.Ca & 0.44 & -0.22 & -0.11 & -0.39 & 0.34 & 0.40 & -0.20 & 0.38 & 0.43 & 0.77 \\
\hline ex.Mg & -0.13 & -0.14 & 0.20 & 0.69 & 0.09 & 0.54 & 0.84 & 0.02 & 0.35 & 0.10 \\
\hline ex. $\mathrm{Na}$ & -0.28 & 0.15 & -0.05 & -0.03 & -0.14 & 0.84 & -0.05 & -0.01 & 0.28 & 0.93 \\
\hline ex.K & -0.10 & 0.14 & 0.03 & 0.85 & -0.05 & -0.09 & -0.72 & -0.03 & 0.63 & 0.14 \\
\hline $\mathrm{Al}$ & 0.17 & 0.36 & -0.10 & 0.61 & 0.40 & -0.14 & 0.54 & -0.34 & 0.30 & -0.43 \\
\hline$P$ & -0.08 & -0.35 & 0.20 & -0.56 & 0.28 & -0.26 & 0.01 & 0.87 & -0.26 & -0.37 \\
\hline K & 0.54 & 0.38 & -0.28 & 0.03 & 0.35 & -0.06 & 0.02 & -0.04 & 0.83 & -0.15 \\
\hline \multicolumn{7}{|c|}{ Component 1: SOC, CEC, ex. Ca, K } & \multicolumn{4}{|c|}{ Component 1: Clay, sand, silt, ex. K, Al } \\
\hline \multicolumn{7}{|c|}{ Component 2: Clay, sand, $\mathrm{pH}$} & \multicolumn{4}{|c|}{ Component 2: Clay, BD, porosity, P } \\
\hline \multicolumn{7}{|c|}{ Component 3: BD, porosity } & \multirow{2}{*}{\multicolumn{4}{|c|}{$\begin{array}{l}\text { Component 3: SOC, CEC, ex. Ca, ex. K, K } \\
\text { Component 4: pH, ex. Ca, ex. } \mathrm{Na}, \mathrm{Al}\end{array}$}} \\
\hline \multicolumn{7}{|c|}{ Component 4: ex. Mg, ex. K, Al, P } & & & & \\
\hline \multicolumn{7}{|c|}{ Component 5: Sand, silt, Al } & & & & \\
\hline \multicolumn{7}{|c|}{ Component 6: pH, ex. Ca, ex. $\mathrm{Mg}$, ex. $\mathrm{Na}$} & & & & \\
\hline
\end{tabular}

*Selected values correspond to the values $>0.5$ or $>0.5$. BD: Bulk density; SOC: Soil organic carbon; CEC: Cation-exchange capacity; Ex.Ca: exchangeable calcium; Ex. Mg: exchangeable magnesium; Ex.K: exchangeable potassium; Al: Aluminum; P: Phosphorus; K: Potassium.

${ }^{\star}$ Bold and underlined factors identified the indicators retained in the MDS and used to calculate SQI.

Table 8. Mean additive soil quality index $\left(\mathrm{SQ} \mathrm{I}_{\mathrm{a}}\right)$ values for different land uses sites at two different soil depths

\begin{tabular}{|l|c|c|}
\hline \multirow{2}{*}{ Land use } & \multicolumn{2}{|c|}{ Additive index (Mean \pm SD) } \\
\cline { 2 - 3 } & \multicolumn{2}{|c|}{ Depth [cm] } \\
\cline { 2 - 3 } & $0-15$ & $15-30$ \\
\hline Plantation $(n=29)$ & $3.29( \pm 0.44)_{b}$ & $2.15( \pm 0.54)_{a}$ \\
Jhum $(n=18)$ & $3.51( \pm 0.51)_{b}$ & $2.52( \pm 0.34)_{b}$ \\
Fallow Jhum $(n=18)$ & $2.13( \pm 0.68)_{a}$ & $3.01( \pm 0.50)_{c}$ \\
Forest $(n=22)$ & $3.27( \pm 0.49)_{b}$ & $2.18( \pm 0.36)_{a}$ \\
\hline
\end{tabular}

*Values followed by the same letter in each columns are not significantly different at $p=0.05$ (Duncan's multiple-range test). 


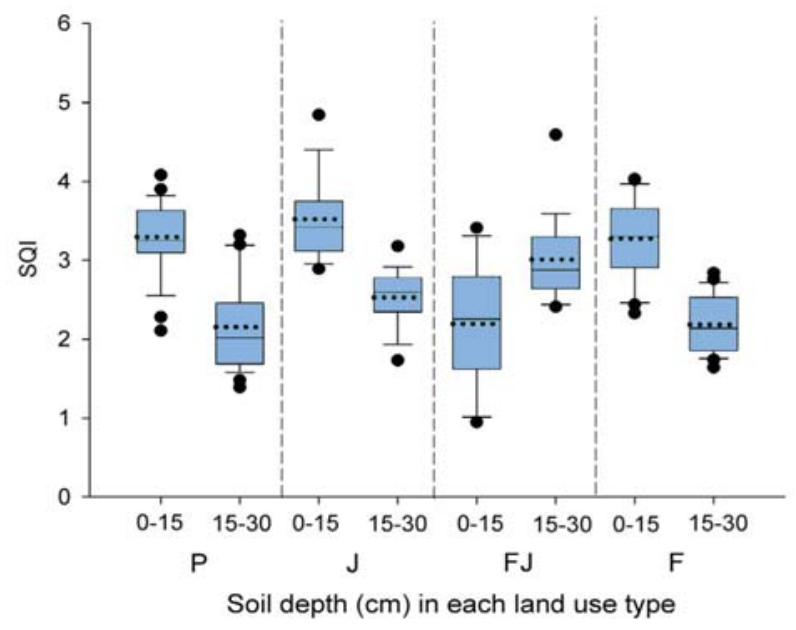

Figure 3. Soil quality index (SQI) values for different land uses sites at two different soil depths

\section{Discussion}

Soil quality assessment and the relationships among soil properties and quality indicators are vital to understanding how humans have to manage their soil resource (Armenise et al., 2013). Therefore, this paper can be considered as one of the first approaches to give new insights about information for developing suitable land management plans or tools to apply effective nature based-solutions for rural inhabitants. However, we acknowledge that further work in this area is much needed yet as other authors in this region also demonstrated (Singh et al., 2014; Lenka et al., 2017), for example, by increasing the soil sample number or analyzing soil properties during different seasons.

Regarding our results, we observed that the mean clay content for the surface layers was higher for Jhum land than for other land uses, which may be attributed due to the shift from forested area to cultivable ones and further soil particle breakdown (Iticha et al., 2016). Moreover, a drastic significant increase in sand content from $15 \mathrm{~cm}$ coincided with the similar findings obtained by Rao and Wagenet (1985), who reported that differences in soil texture with increasing soil depth because of the high weathering ratios, erosion-deposition dynamics and soil-forming factors. Although there was no significant difference in BD values, an increase in values along the depth was noticed in all the land uses, which can be explained by the effects of soil compaction. As Bogunovic et al., (2017) reported, after transform forestry areas with Stagnosols into vineyards, soil compaction measured by soil penetration resistance and bulk density confirmed a drastically increase at the subsurface layers. Also, Williams and Brevik (2010) found in sandy soils this effect due to the tractor passes in South Georgia. On the contrary, in Mediterranean areas, after the abandonment and vegetation recovery, Bienes et al., (2016) observed that bulk density decreased due to the reduction of tillage practices and trampling effect. In forest soils, a reverse trend in $\mathrm{BD}$ was also observed. Moreover, this variation in BD values with elevation (Hanawalt \& Whittaker, 1976) and its negative correlation with organic matter (Sharma et al., 2010) is already well defined. Regarding the porosity values, the results also showed a decrease in-depth for all land uses and differences were non-significant, except for fallow lands, where the significant decrease was observed. This can be supported with results of Deuchars et al. (1999), 
who reported that due to the conversion from forest land into pastures or agricultural lands, soils became compact (coinciding with the increase in BD) and their porosity will subsequently decrease. Similarly, Kizilkaya and Dengiz (2010) also reported that cultivation leads to compaction and a decrease in porosity. The soils of this region are highly acidic due to leaching of bases from the exchange complex under prevailing high rainfall and hilly topography (Singh et al., 2014), so no significant difference was observed in the $\mathrm{pH}$ values at different soil depths and land uses.

SOC content was higher in the surface of all land uses and an inverse relationship with the BD values except for forests. We hypothesize that it is due to the high amount of litterfall and slow decomposition of organic matter in forest areas (Agren et al., 2013; Mishra et al., 2017). Moreover, SOC content was significantly influenced by soil depth in plantation, fallow Jhum lands and forests. There are several studies throughout the world indicating a decrease of SOC from forest to grassland and cropland (Powlson et al., 2011; Don et al., 2011). However, we could not confirm these general results from our finding as for the surface soil $(0-15 \mathrm{~cm})$ layer, SOC contents were fallow Jhum > plantation> Jhum > forest. These results are in the line with Mendoza-Vega and Messing, (2005), who reported that a soil recovery process took place in fallow lands and an increase over the time in SOC can be recorded. Similarly, Sarkar et al., (2015) also reported a significant rise in SOC with the increase in the fallow period.

CEC was strongly influenced by physical (e.g., texture) and chemical (e.g., pH and SOC) properties (Khaledian et al., 2017). Accordingly, in our study also, CEC is found to decrease in-depth for all land uses, but a significant difference was noticed only in fallow Jhum land soils. Brady and Weil (2008) also reported that SOM, which is positively correlated with SOC, has the highest CEC values. Our results are also in the line of these findings, as fallow Jhum land soils showed a significant decrease in SOC values with an increase in soil depth, and subsequently, in CEC too. Also, Zeraatpishe and Khormali, (2012) reported that SOC can affect the $\mathrm{pH}$ values, as CEC in Iran. In SE Asia, Bruun et al., (2009) observed clear impacts of swidden cultivation on carbon storage and soil quality considering the intensification from the traditional production systems to the modern ones. They highlighted that the time-averaged aboveground carbon stocks decline depending on the reduction of about 4 or 6 years in fallow periods of traditional swidden cultivation the transformation or into oil palm plantations, respectively. We also coincide that the effects of soil management is fundamental to understand soil quality changes.

Among the Ex. cations, only $\mathrm{Ca}^{2+}$ and $\mathrm{K}^{+}$, showed some significant differences among soil depths, in the plantation, Jhum and fallow Jhum soils. Vasu et al., (2016) also reported that the variations in clay content, SOC, Ex. cations and CEC may be attributed to a combination of intrinsic (weathering, erosion, deposition and soil-forming processes) and extrinsic (management practices) factors.

In this way, PCA allows us to select different soil quality indicators for each different land use. Our results, mainly soil texture, $\mathrm{BD}$, porosity, SOC and ex cations were come up as potential indicators of soil quality. These findings were consistent with earlier studies of Brejda and Moorman (2001), Cho et al. (2004) and Shukla et al. (2006), who also reported that soil texture, can be considered as an important indicator in soil quality assessment. Moreover, Vinhal-Freitasa et al. (2017) reported that soil textural classes are of great importance in evaluating soil functions and quality in tropical ecosystems like our study area, NE India. Importance of other physical properties like BD and porosity as SQ indicator was already established by several researchers, as they play an important role in the regulation of water transmission, root penetration, retention of nutrients and water, soil erosion and runoff (Al-Shammary et al., 2018). Similarly, the importance of SOC as a self-sufficient soil quality indicator is advocated by Lal (2002), as it plays an important 
role in nutrient supply, soil moisture retention and soil aggregate stability. Soil chemical properties such as cation exchange capacity and extractable anion and cations have all been used as effective chemical indicators of soil quality. In all the land uses, their values were found to decrease with the depth due to its correspondence with the clay content (Bhaskar et al., 2005; Khan \& Kamalkar, 2012).

Deriving SQI, using PCA and MDS from a specific number of parameters, is a useful approach to evaluate land uses in terms of its sustainability. The soil quality of the studied district was strongly influenced by the land use and soil depth. In topsoil (0-15cm), Jhum soils had the best SQI in comparison to other land uses. Although, no significant differences were observed among the SQI value of fallow Jhum land, forest and plantation soils. In Jhum lands, before the cultivation, secondary vegetation was slashed and burned in fields, which may increase the availability of nutrients ( $\mathrm{P}, \mathrm{K}, \mathrm{Ca}$ and $\mathrm{Mg}$ ) in surface layers (Lungmuana et al., 2018), increasing the results of SQI. Moreover, the burning of plant biomass and subsequent release of alkaline cations can increase soil pH (Dikici \& Yilmaz, 2006), and also contributes to maintaining the availability of nutrients. Reports from other parts obtained similar results (Singh et al., 2014 and Mishra et al., 2017), which are supporting better soil quality in Jhum lands. This improved status of soils boosts the crop growth in 1-2 year of cultivation, but a decline in subsequent years due to cultivation, leaching, runoff and erosion (Tawnenga et al., 1997). Jhum lands were converted into fallow Jhum lands with degraded quality as reported in this study. Soil erosion is another recognized issue related to these types of land use transformation and changes in management systems. Gafur et al., (2003) reported that soil erosion under fallow lands can usually reach 3 $\mathrm{Mg} \mathrm{ha}^{-1} \mathrm{yr}^{-1}$. However, during the cultivation year, this amount could even exceed six times as high, which is particularly unsustainable, considering the tolerable soil erosion rates mentioned by other authors in the past (Verheijen et al., 2009).
Forest and plantation soils had less value of SQI in comparison to cropland, which can be supported with the well-known fact that, much of the nutrient reserve in tropical regions is stored in plant tissue, but critical nutrient limitation will occur, if biomass is removed (Dalling et al., 2016). A higher value of SQI in fallow Jhum lands at $15-30 \mathrm{~cm}$ soil depth may be attributed due to the fast growth of the secondary succession vegetation during the fallow phases. However, as other authors mentioned in India, these soil responses significantly can vary at different spatial scales due to the slope inclination, parent material and aspect (Prokop et al., 2018).

This types of research can also have a relevant impact related to human population growth and the impacts of land-use changes on food security (Brevik et al., 2020, 2019). This is a novel aspect highlighted by the pioneer and recently study conducted by Behera et al. (2016). They considered that the food production, diversity and quality was more elevated in the Jhum lands and traditional cash-cropping compared to any of the modern cash-crop systems. In a literature review, Grogan et al., (2012) recommended some possibilities for improving shifting cultivation in India. The main ones are related to nutrient and water supplementation, optimizing crop choice, extending the site use period, enhancing the fallow recovery rate, and controlling the burns and their environmental impacts. They also suggest the use of inter-row cropping between contour hedgerows, the use of terraces or vegetation cover to reduce soil erosion. These are not new methods that are highly implemented in other vulnerable territories such as the Mediterranean belt or countries such as Iran or China affected by soil pollution, erosion or nutrient depletion (e.g. Durán Zuazo et al., 2011; Novara et al., 2013; Yu et al., 2017; Yazdanbakhsh et al., 2020).

\section{Conclusion}

Land-use changes are well known to be a driving factor of physical, chemical and biological 
soil variations. In this study, the soil quality of four different sequential land uses worked by traditional farmers groups in the Himalayas were evaluated, at two soil depths. Our main findings confirmed that Jhum soils obtained the best soil quality at the surface layer. At the sub-surface, the highest soil quality index result was obtained for the fallow Jhum soils. We claim that soil quality in this region can be resumed in a specific set of soil properties. Therefore, data reduction techniques such as PCA and SQI were confirmed as techniques to identify soil quality indicators. Using these indicators, we were able to evaluate soil quality being more productive in terms of time- and money- consuming.

\section{Editors' note:}

Unless otherwise stated, the sources of tables and figures are the authors', on the basis of their own research.

\section{References}

Abdel Rahman, M.A.E., Shalaby, A., Mohamed, E.S. (2019). Comparison of two soil quality indices using two methods based on geographic information system. The Egyptian Journal of Remote Sensing and Space Science, 22, 127-136. https://doi.org/10.1016/j.ejrs.2018.03.001

Agren, G.I., Hyvonen, R., Berglund, S.L., Hobbie, S.E. (2013). Estimating the critical N: C from litter decomposition data and its relation to soil organic matter stoichiometry. Soil Biology and Biochemistry, 67, 312-318. https://doi.org/10.1016/j.soilbio.2013.09.010

Al-Shammary, A.A.G., Kouzani, A.Z., Kaynak, A., Khoo, S.Y., Norton, M., Gates, W. (2018). Soil bulk density estimation methods: A review. Pedosphere, 28, 581-596. https://doi.org/10.1016/S1002-0160(18)60034-7

Armenise, E., Redmile-Gordon, M.A., Stellacci, A.M., Ciccarese, A., Rubino, P. (2013). Developing a soil quality index to compare soil fitness for agricultural use under different managements in the Mediterranean environment. Soil and Tillage Research 130, 91-98. https://doi.org/10.1016/j.still.2013.02.013

Arunachalam, A. (2002). Dynamics of soil nutrients and microbial biomass during first year cropping in an 8-year jhum cycle. Nutrient Cycling in Agroecosystems, 64, 283-291. https://doi.org/10.1023/A:1021488621394

Bahr, E., Zaragocin, D.C., Makeschin, F. (2014). Soil nutrient stock dynamics and land-use management of annuals, perennials and pastures after slash-and-burn in the Southern Ecuadorian Andes. Agriculture, Ecosystems \& Environment, 188, 275-288. https://doi.org/10.1016/j.agee.2014.03.005

Behera, R.N., Nayak, D.K., Andersen, P., Måren, I.E. (2016). From jhum to broom: Agricultural land-use change and food security implications on the Meghalaya Plateau, India. Ambio, 45(1), 63-77. https://doi.org/10.1007/s13280-015-0691-3

Beniston, J.W., Lal, R., Mercer, K.L. (2015). Assessing and managing soil quality for urban agriculture in a degraded vacant lot soil. Land Degradation \& Development, 27(4), 996-1006. https://doi.org/10.1002/ldr.2342

Bhaskar, B.P., Butte, P.S., Utpal Brauah, S. (2005). Characterization of soils in the 'Bil' environs of Brahmaputra valley in Jorhat district, Assam for land use interpretation. Journal of Indian Society of Soil Science, 53, 3-10. Retrieved from https://www.indianjournals.com/ijor.aspx?target=ijor:jisss\&volume=53\&issue=1\&article=001

Bienes, R., Marques, M.J., Sastre, B., García-Díaz, A., Ruiz-Colmenero, M. (2016). Eleven years after shrub revegetation in semiarid eroded soils. Influence in soil properties. Geoderma, 273, 106-114. https://doi.org/10.1016/j.geoderma.2016.03.023

Blake, G.R., Hartge, K.H. (1986). Bulk density. In Klute, A. (Ed.), Methods of Soil Analysis: Part 1, Physical and Mineralogical Methods, 5.1, Second edition (pp. 951-984). Madison, Wisconsin, USA: Soil Science Society of America. https://doi.org/10.2136/sssabookser5.1.2ed.c13 
Bogunovic, I., Bilandzija, D., Andabaka, Z., Stupic, D., Rodrigo-Comino, J., Cacic, M., Brezinscak, L., Maletic, E., Pereira, P. (2017). Soil compaction under different management practices in a Croatian vineyard. Arabian Journal of Geosciences, 10, 340. https://doi.org/10.1007/s12517-017-3105-y

Bray, RH., Kurtz, LT. (1945). Determination of total, organic and available forms of phosphorus in soils. Soil Science, 59, 39-46.

Brady, NC., Weil, RR. (2008). Nature and properties of soils. 14th Ed. Upper Saddle River, NJ: Prentice Hall.

Brevik, E.C., Slaughter, L., Singh, B.R., Steffan, J.J., Collier, D., Barnhart, P.,Pereira, P. (2020). Soil and human health: Current status and future needs. Air, Soil and Water Research, 13. https://doi.org/10.1177/1178622120934441

Brevik, E.C., Steffan, J.J., Rodrigo Comino, J., Neubert, D., Burgess, L.C., Cerdà, A. (2019). Connecting the public with soil to improve human health. European Journal of Soil Science, 70(4), 898-910. https://doi.org/10.1111/ejss.12764

Bruun, T.B., de Neergaard, A., Lawrence, D., Ziegler, A.D. (2009). Environmental consequences of the demise in Swidden cultivation in Southeast Asia: Carbon storage and soil quality. Human Ecology, 37(3), 375-388. https://doi.org/10.1007/s10745-009-9257-y

Brejda, J.J., Moorman, T.B. (2001). Identification and interpretation of regional soil quality factors for the central high plains of the Midwestern USA. In: D.E. Stott, R.H. Mohtar and G.C. Steinhardt (Eds.). Sustaining the global farm (pp. 535-540). Selected papers from the 10th International Soil Conservation Organisation Meeting held during May 24-29 at Purdue University and the USDA-ARS National Soil Erosion Research Laboratory

Chase, P., Singh, O.P. (2014). Soil nutrients and fertility in three traditional land use systems of Khonoma, Nagaland, India. Resources and Environment, 4, 181-189. https://doi.org/10.5923/j.re.20140404.01

Cho, K.M., Zoebisch, M.A., Ranamukhaarachchi, S.L. (2004). Land-use dependent soil quality in the Lam Phra Phloeng watershed, northeast Thailand. Paper no 119. In 13th International Soil Conservation Organisation Conference on "Conserving Soil and Water for Society: Sharing Solutions" held in Brisbane, July 2004.

Chen, C., Liu, W., Jiang, X., Wu, J. (2017). Effects of rubber-based agroforestry systems on soil aggregation and associated soil organic carbon: Implications for land use. Geoderma, 299, 13-24. https://doi.org/10.1016/j.geoderma.2017.03.021

Choudhury, B.U., Fiyaz, A.R., Mohapatra, K.P., Ngachan, S. (2016). Impact of land uses, agrophysical variables and altitudinal gradient on soil organic carbon concentration of North Eastern Himalayan Region of India. Land Degradation \& Devlopment, 27, 1163-1174. https://doi.org/10.1002/Idr.2338

Dalling, J.W., Heineman, K., Lopez, O.R., Wright, S.W., Turner, B.L. (2016). Nutrient Availability in Tropical Rain Forests: The Paradigm of Phosphorus Limitation. In G. Goldstein and L.S. Santiago (Eds.), Tropical Tree Physiology (pp. 261-273). https://doi.org/10.1007/978-3-319-27422-5_12

Deuchars, S.A, Townend, J., Aitkenhead, M.J., Fitzpatrick, E.A. (1999). Changes in structure and hydraulic properties in regenerating rain forest. Soil Use and Management, 15, 183-187. https://doi.org/10.1111/j.1475-2743.1999.tb00086.x

Dikici, H., Yilmaz, C.H. (2006). Peat fire effects on some properties of an artificially drained peatland. Journal of Environmental Quality. 35, 866-870. DOI 10.2134/jeq2005.0170.

Don, A., Schumacher, J., Freibauer, A. (2011). Impact of tropical land-use change on soil organic carbon stocks - a meta-analysis. Global Change Biol. 17, 1658-1670. https://doi.org/ 10.1111/j.1365- 2486.2010.02336.x

Durán Zuazo, V.H., Rodríguez Pleguezuelo, C.R., Martin Peinado, F.J., de Graaff, J., Francia Martínez, J.R., Flanagan, D.C. (2011). Environmental impact of introducing plant covers in the taluses of terraces: Implications for mitigating agricultural soil erosion and runoff. Catena, 84(1-2), 79-88. https://doi.org/10.1016/j.catena.2010.10.004

FSI (Forest Survey of India). (2009). State of the Forest Report. Dehradun: FSI. 
Gafur, A., Jensen, J.R., Borggaard, O.K., \& Petersen, L. (2003). Runoff and losses of soil and nutrients from small watersheds under shifting cultivation (Jhum) in the Chittagong Hill Tracts of Bangladesh. Journal of Hydrology, 274(1), 30-46. https://doi.org/10.1016/S0022-1694(02)00351-7

Grogan, P., Lalnunmawia, F., Tripathi, S.K. (2012). Shifting cultivation in steeply sloped regions: A review of management options and research priorities for Mizoram state, Northeast India. Agroforestry Systems, 84(2), 163-177. https://doi.org/10.1007/s10457-011-9469-1

Hanawalt, R.B., Whittaker, R.H. (1976). Attitudinally coordinated patterns of soils and vegetation in-the San Jacinto Mountains. California. Soil Science, 121, 114-124.

Handayani, I.P. (2004). Soil quality changes following forest clearance in Bengkulu, Sumatra. Biotropia, 22, 15-28.

Iticha, B., Mohammed, M., Kibret, K. (2016). Impact of deforestation and subsequent cultivation on soil fertility in Komto, Western Ethiopia. Journal of Soil Science and Environmental Management. 7(12), 212-221. https://doi.org/10.5897/JSSEM2016.0578

IPCC. (2007). In Bernstein, L., Bosch, P., Canzioni, O., et al. (Eds.), Climate Change 2007: Synthesis report. An assessment of the intergovernmental panel on climate change. IPCC, Valencia, Spain. https://archive.ipcc.ch/publications_and_data/publications_and_data_reports.shtml

Keshavarzi, A., Tuffour, H., Bagherzadeh, A., Tattrah, L.P., Kumar, V., Gholizadeh, Rodrigo-Comino, J. (2020). Using fuzzy-AHP and parametric technique to assess soil fertility status in Northeast of Iran. Journal of Mountain Science, 17(4), 931-948. https://doi.org/10.1007/s11629-019-5666-6

Khaledian, Y., Kiani, F., Ebrahimi, S., Brevik, E.C., Aitkenhead-Peterson, J. (2017). Assessment and monitoring of soil degradation during land use change using multivariate analysis. Land Degradation \& Development, 28, 128-141. https://doi.org/10.1002/ldr.2541.

Khan, MA., Kamalakar, J. (2012). Physical, physico-chemical and chemical properties of soils of newly established Agro-biodiversity Park of Acharya NG Ranga Agricultural University, Hyderabad, Andhra Pradesh. International Journal of Farm Sciences, 2(2), 102-116.

Klute, A. (Ed.). (1986). Methods of soil analysis: Part 1. Physical and Mineralogical Methods. Soil Science Society of America Book Series No. 5. Madison, Wisconsin: Soil Science Society of America.

Kizilkaya, R., Dengiz, O. (2010). Variation of land use and land cover effects on some soil physico-chemical charactersitcs and soil enzyme activity. Zemdirbyste-Agriculture, 97(2), 15-24.

Lal, R. (2002).Why carbon sequestration in agricultural soils? In: Kimble, J.M., Lal, R., Follet, R.F. (Eds.), Agricultural practices and policies for carbon sequestration in soil (pp. 21-30). Boca Raton, FL, USA: Lewis Publications.

Lenka, N.K., Satapathy, K.K., Lal, R., Singh, R.K., Singh, N.A.K., Agrawal, P.K., Choudhury, P., Rathore, A. (2017). Weed strip management for minimizing soil erosion and enhancing productivity in the sloping lands of north-eastern India. Soil and Tillage Research, 170, 104-113. https://doi.org/10.1016/j.still.2017.03.012

Lohbeck, M., Poorter, L., Martínez-Ramos, M., Bongers, F., (2015). Biomass is the main driver of changes in ecosystem process rates during tropical forest succession. Ecology, 96, https://doi.org/10.1890/14-0472.1

Lungmuana, S., Choudhury, B.U., Saha, S., Singh, S.B., Das, A., Buragohain, J., ... Dutta, S.K. (2018). Impact of postburn jhum agriculture on soil carbon pools in the north-eastern Himalayan region of India. Soil Research, 56(6), 615-622. https://doi.org/10.1071/SR18031

McLean, E.O. (1965). Aluminum. In: Black, C.A. (Ed.) Methods of soil analysis: Part 2. Chemical methods (pp. 978-998). Madison: ASA. https://doi.org/10.2134/agronmonogr9.2.c16

Mendoza-Vega, J., Messing, I. (2005). The influence of land use and fallow period on the properties of two calcareous soils in the humid tropics of southern Mexico. Catena, 60, 279-292.

https://doi.org/10.1016/j.catena.2004.12.002 
Mishra, G., Das, P.K., Borah, R., Dutta, A. (2017). Investigation of phytosociological parameters and physico-chemical properties of soil in tropical semi-evergreen forests of Eastern Himalaya. Journal of Forestry Research, 28, 513-520. https://doi.org/10.1007/s11676-016-0330-2

Mishra, G., Marzaioli, R., Giri, K., Borah, R., Dutta, A., Jayaraj, R.S.C. (2017). Soil quality assessment under shifting cultivation and forests in Northeastern Himalaya of India. Archives of Agronomy and Soil Science, 63, 1355-1368. https://doi.org/10.1080/03650340.2017.1281390

Mukherjee, A., Lal, R. (2014). Comparison of soil quality index using three methods. PLOS ONE, 9, e105981. https://doi.org/10.1371/journal.pone.0105981

Mulugeta, L., Karltun, E., Olsson, M. (2005). Assessing soil chemical and physical property responses to deforestation and subsequent cultivation in smallholders farming system in Ethiopia. Agriculture, Ecosystems \& Environment, 105, 373-386. https://doi.org/10.1016/j.agee.2004.01.046

Nega, E., Heluf, G. (2009). Influence of land use changes and soil depth on cation exchange capacity and contents of exchangeable bases in the soils of Senbat Watershed, western Ethiopia. Ethiopian Journal of Natural Resources, 11, 195-206.

Norman, L.M. (2020). Ecosystem Services of Riparian Restoration: A Review of Rock Detention Structures in the Madrean Archipelago Ecoregion. Air, Soil and Water Research, 13, 1178622120946337. https://doi.org/10.1177/1178622120946337

Novara, A., Gristina, L., Guaitoli, F., Santoro, A., Cerdà, A. (2013). Managing soil nitrate with cover crops and buffer strips in Sicilian vineyards. Solid Earth, 4(2), 255-262. https://doi.org/10.5194/se-4-255-2013

Ozan, D.Y., Omar, A., Gurdal, T. (2008). Multivariate statistics to investigate metal contamination in surface soil. Journal of Environmental Management, 86, 581-594. https://doi.org/10.1016/j.jenvman.2006.12.032

Palm, A.A., Swift, M.J., Woomer, P.L. (1996). Soil biological dynamics in slash-and-burn agriculture. Agriculture, Ecosystems \& Environment, 58, 61-74. https://doi.org/10.1016/0167-8809(95)00653-2

Patel, T., Karmakar, S., Sanjog, J., Kumar, S., Chowdhury, A. (2013). Socio-economic and environmental changes with transition from shifting to settled cultivation in North-Eastern India: an ergonomics perspective. International Journal of Agricultural Science and Research. 3, 117-136.

Poorter, L., van der Sande, M.T., Thompson, J., Arets, E.J., Alarcón, A., Álvarez Sanchez, J., ... Peña Claros, M. (2015). Diversity enhances carbon storage in tropical forests. Global Ecology and Biogeography, 24(11): https://doi.org/10.1111/geb.12364

Powlson, D.S., Whitmore, A.P., Goulding, K.W.T. (2011). Soil carbon sequestration to mitigate climate change: A critical re-examination to identify the true and the false. European Journal of Soil Science, 62, 42-55. https://doi.org/10.1111/j.1365-2389.2010.01342.x

Prokop, P., Kruczkowska, B., Syiemlieh, H.J., Bucała-Hrabia, A. (2018). Impact of topography and sedentary swidden cultivation on soils in the hilly uplands of North-East India. Land Degradation \& Development, 29(8), 2760-2770. https://doi.org/10.1002//dr.3018

Ramakrishnan, P.S., Toky, O.P. (1981). Soil nutrient status of hill agroecosystems and recovery pattern after slash and burn agriculture (jhum) in north-eastern India. Plant and Soil, 60, 41-64. https://doi.org/10.1007/BF02377111

Rao, P.S.C., Wagenet, R.J. (1985). Spatial variability of pesticides in field soils: Methods for data analysis and consequences. Weed Science, 33, 18-24. https://doi.org/10.1017/S0043174500083764

Rodrigo-Comino, J., Keshavarzi, A., Bagherzadeh, A., Brevik, E.C. (2019). The use of multivariate statistical analysis and soil quality indices as tools to be included in regional management plans. A case study from the Mashhad Plain, Iran. Cuadernos de Investigación Geográfica, 45, 687-708. https://doi.org/10.18172/cig.3640

Saha, R., Chaudhary, R.S., Somasundaram, J. (2012). Soil health management under hill agroecosystem of North East India. Applied and Environmental Soil Science, 2012, 1-9.

https://doi.org/10.1155/2012/696174 
Salehi, A., Wilhelmsson, E., Soderberg, U. (2008). Land cover changes in a forested watershed, southern Zagros, Iran. Land Degradation \&. Development, 19, 542-553. https://doi.org/10.1002/ldr.860

Sarkar, D., Meitei, Ch.B., Baishya, L.K., Das, A., Ghosh, S., Chongloi, K.L., Rajkhowa, D.J. (2015). Potential of fallow chronosequence in shifting cultivation to conserve soil organic carbon in northeast India. Catena, 135, 321-327. https://doi.org/10.1016/j.catena.2015.08.012.

Schollenberger, C.J., Simon, R.H. (1945). Determination of exchange capacity and exchangeable bases in soil. Ammonium acetate method. Soil Science, 59, 13-24.

Sharma, C.M., Gairola, S., Ghildiyal, S.K., Suyal, S. (2010). Physical properties of soils in relation to forest composition in moist temperate valley slopes of the Central Western Himalaya. Journal of Forest and Environmental Science, 26(2), 117-129.

Shukla, M.K., Lal, R., Ebinger, M. (2006). Determining soil quality indicators by factor analysis. Soil Tillage Research, 87, 194-204. https://doi.org/10.1016/j.still.2005.03.011

Singh, N.P., Singh, O.P., Jamir, N.S. (1996). Sustainable agriculture development strategy for North Eastern Hill Region of India. In S.P. Shukla, N. Sharma (Eds.), Sustainable development strategy (Indian context) (pp.346-351). New Delhi: Mittal.

Singh, A.K., Bordoloi, L.J., Kumar, M., Hazarika, S., Parmar, B. (2014). Land use impact on soil quality in eastern Himalayan region of India. Environmental Monitoring and Assessment, 186, 2013-2024. https://doi.org/10.1007/s10661-013-3514-7

Sulieman, M., Saeed, I., Hassaballa, A., Rodrigo-Comino, J. (2018). Modeling cation exchange capacity in multi geochronological-derived alluvium soils: An approach based on soil depth intervals. Catena, 167, 327-339. https://doi.org/10.1016/j.catena.2018.05.001

Sumner, M.E., Miller, W.P. (1996). Cation exchange capacity and exchange coefficients. In D.L. Sparks, A.L. Page, P.A. Helmke (Eds.), Methods of Soil Analysis Part 3, Chemical Methods (pp. 1201-1229). Madison, Wisconsin, USA: Soil Science Society of America.

Tawnenga, Shankar, U., Tripathi, R.S. (1997). Evaluating second year cropping on jhum fallows in Mizoram, north-eastern India: soil fertility. Journal of Biosciences, 22, 615-625.

https://doi.org/10.1007/BF02703399

USDA Natural Resources Conservation Service. (2004). In R. Burt (Ed.), Soil survey laboratory methods manual. Soil Survey Investigations Report No. 42, Version 4.0. Lincoln, NE: National Soil Survey Center.

Vasu, D., Singh, S.K., Ray, S.K, Duraisami, V.P, Tiwary, P., Chandran, P., Nimkar, A.M., Anantwar, S.G. (2016). Soil quality index (SQI) as a tool to evaluate crop productivity in semi-arid Deccan plateau, India. Geoderma, 282, 70-79. https://doi.org/10.1016/j.geoderma.2016.07.010

Verheijen, F.G.A., Jones, R.J.A., Rickson, R.J., \& Smith, C.J. (2009). Tolerable versus actual soil erosion rates in Europe. Earth-Science Reviews, 94(1-4), 23-38. https://doi.org/10.1016/j.earscirev.2009.02.003

Vorlaufer, T., Falk, T., Dufhues, T., Kirk, M. (2017). Payments for ecosystem services and agricultural intensification: Evidence from a choice experiment on deforestation in Zambia. Ecological Economics, 141, 95-105. https://doi.org/10.1016/j.ecolecon.2017.05.024

Vinhal-Freitasa, I.C., Corrêa, G.F., Wendling, B., Bobul'ská, L., Ferreira, A.S. (2017). Soil textural class plays a major role in evaluating the effects of land use on soil quality indicators. Ecological Indicators, 74, 182-190. https://doi.org/10.1016/j.ecolind.2016.11.020

Walkley, A., Black, I.A. (1934). An examination of the Degtjareff method for determining soil organic matter and a proposed modification of the chromic acid titration method. Soil Science, 37(1), 29-38.

Williams, M., Brevik, E.C. (2010). Effect of traffic rate and type on soil compaction in sandy South Georgia soils. Soil Horizons, 51. https://doi.org/10.2136/sh2010.3.0088

Yadav, P.K. (2013). Slash-and-burn agriculture in north-East India. Expert Opinion on Environmental Biology, 2, 1-4. https://doi.org/10.4172/2325-9655.1000102 
Yazdanbakhsh, A., Alavi, S.N., Valadabadi, S.A., Karimi, F., Karimi, Z. (2020). Heavy metals uptake of salty soils by ornamental sunflower, using cow manure and biosolids: A case study in Alborz city, Iran. Air, Soil and Water Research, 13, 1178622119898460. https://doi.org/10.1177/1178622119898460

Yu, Y., Wei, W., Chen, L., Feng, T., Daryanto, S., Wang, L. (2017). Land preparation and vegetation type jointly determine soil conditions after long-term land stabilization measures in a typical hilly catchment, Loess Plateau of China. Journal of Soils and Sediments, 17(1), 144-156. https://doi.org/10.1007/s11368-016-1494-2

Zeraatpishe, M., Khormali, F. (2012). Carbon stock and mineral factors controlling soil organic carbon in a climatic gradient, Golestan Province. Journal of Soil Science and Plant Nutrition, 12(4), 637-654. https://doi.org/10.4067/S0718-95162012005000022 